\title{
Filtering of Biomedical signals by using Complete Ensemble Empirical Mode Decomposition with Adaptive Noise
}

\author{
S. Elouaham \\ Laboratory of Systems \\ Engineering and Information \\ Technology (LiSTi) \\ National School of Applied \\ Sciences, Ibn Zohr University \\ Agadir, Morocco
}

\author{
A. Dliou \\ Laboratory of Systems \\ Engineering and Information \\ Technology (LiSTi), \\ National School of Applied \\ Sciences, Ibn Zohr University \\ Agadir, Morocco
}

\author{
R. latif, M. Laaboubi \\ Laboratory of Systems \\ Engineering and Information \\ Technology (LiSTi) \\ National School of Applied \\ Sciences, Ibn Zohr University \\ Agadir, Morocco
}

\begin{abstract}
This work treats the filtering of artifacts that interfered with the ECG signals by the different denoising methods for ameliorate the reliability accuracy. During ECG measurement, there may be various noises such as muscle contraction (electromyography), baselines wander and power-line interferences, which interfered with the ECG information identification that causing a misinterpretation of the ECG signal. In this paper, the denoising techniques of the Empirical Mode Decomposition (EMD), the Ensemble Empirical Mode Decomposition (EEMD) and the Complete Ensemble Empirical Mode Decomposition with adaptive noise (CEEMDAN) are used. The obtained results of the CEEMDAN technique exceed others methods (EEMD and EMD) used in this paper. The CEEMDAN technique is successful in denoising the biomedical signals.
\end{abstract}

\section{Keywords}

CEEMDAN, EEMD, EMD, CU Ventricular Tachyarrhythmia, Malignant Ventricular.

\section{INTRODUCTION}

The electrocardiogram (ECG) is one of the most important biomedical signals taken from a human body; it provides indirect evidence of blood flow to the heart muscle. It is considered as a heart activity measuring tool. The interpretation of the ECG signal requires significant amounts of training. The figure 1 shows the normal ECG signal [1-2]:

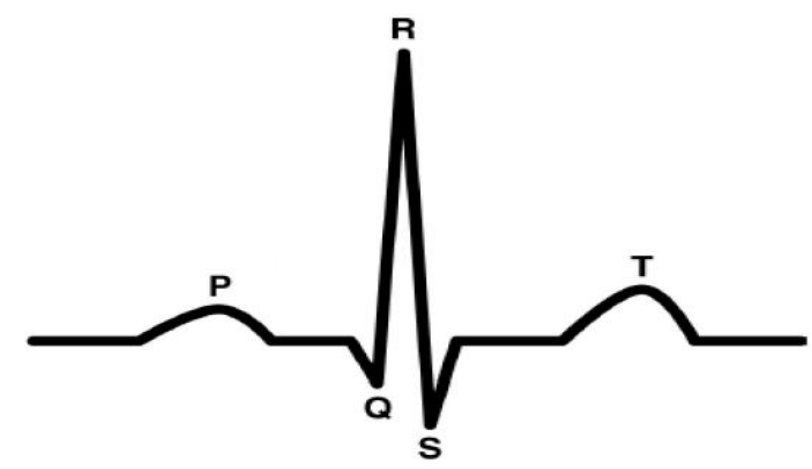

Figure 1: A normal ECG signal.

The ECG signal is used to indicate the condition of the heart; in case of the disorder in heart rate or normal rhythm or change in the morphological pattern of the QRS complex, waveform of the $\mathrm{T}$ and $\mathrm{P}$. However, these irregularities cannot be consistent and hence it can be very difficult even for a trained physician to do a proper diagnosis. The ECG signal can be affected by different types of noise such as prolonged repolarization, changes of electrode position, muscle contraction (electromyography) and power line interference that get embedded with ECG signal during its acquisition and transmission [3]. The reduction of the noise in ECG signals is a very important step during the acquisition or the analysis of electrical activity of the heart. The noise in the normal and abnormal ECG signals can hide the useful information of the important components (waves $\mathrm{P}, \mathrm{T}$ and QRS complex). Among the objectives of this study is to separate the signal component from the undesired artifacts. These artifacts cannot facilitate a good interpretation of the ECG signals; this implies a bad diagnostic given by the expert. Therefore; the elimination of noise is inevitable. To overcome this problem, several techniques, whose goal is to cancel or reduce the noise existing during the recordings of the biomedical signals, are presented

The current study proposes to use the EMD, EEMD and CEEMDAN techniques [3-10]. The traditional filters as Wiener filter are easy to design; most of these techniques are not suitable for analyzing non-stationary and non-linear biological signals (ECG, EEG, EMG...). The disadvantage of EMD is the appearance of mode-mixing effect in signal restoration [3-5]. The EEMD technique is presented to avoid the mode mixing by studying the statistical characteristics of white noise [8]. The disadvantages of this technique are the interaction between signal and noise. The problems of white noise are not totally eliminated. To surpass this problem, the complementary ensemble empirical mode decomposition with adaptive noise (CEEMDAN) is presented. The white noises added in abnormal biomedical signals are negative and positive; the biomedical signal is afterwards decomposed by EEMD. The IMFs of CEEMDAN technique is obtained by using the EEMD technique for abnormal biomedical signals, with adding positive and negative white noise. The choice of a powerful technique among these techniques is related to the result obtained after denoising ECG signals; these results are the original characteristic waveforms such as QRS complexes, the $\mathrm{P}$ and $\mathrm{T}$ waves and also $\mathrm{Q}, \mathrm{R}$ and $\mathrm{S}$ waves.

In this work; we present a complete Ensemble Empirical Mode decomposition with Adaptive Noise (CEEMDAN) technique that gives an exact reconstruction of the original biomedical [11-14]. The CEEMDAN technique gives many promising features for processing biomedical signals 
The paper is organized as follows. Section 2 deals with the denoising techniques. Section 3 provides abnormal electrocardiogram signals. The obtained results are given in section 4 . Finally, conclusion are provided in section 5 .

\section{DENOISING TECHNIQUES USED}

\subsection{EMD}

The EMD was proposed by Huang and al. as a tool to adaptively decompose a signal into a collection of AM-FM components [3]. The EMD method has no mathematical foundations and analytical expressions for the theoretical study. The various works have successfully used the EMD to real data in several fields such as biomedicine, study of climate phenomena, seismology or acoustics [4-5]. These studies show satisfaction and matching condition used in non-stationary signal processing. The EMD decomposes adaptively a nonstationary signal into a sum of functions oscillatory bandlimited $d(t)$ called Intrinsic Mode Functions $\operatorname{IMFJ}(t)$. These functions $\operatorname{IMFJ}(\mathrm{t})$ oscillate around zero and can express the signal $\mathrm{x}(\mathrm{t})$ by the expression:

$$
x(t)=\sum_{j=1}^{k} d_{j}(t)+r(t)
$$

Where $r(t)$ is the residue of low frequency.

Each IMFJ(t) must satisfy two conditions:

The number of zero crossings and the number of extreme signal must be equal throughout the analyzed signal.

At any point, the average of the envelopes defined by local extreme of the signal must be 0 .

The higher order IMFJ(t) corresponds to low oscillation components, while lower-order IMFJ $(t)$ represents fast oscillations. For different decomposed signals the number of $\operatorname{IMFJ}(t)$ is variable. It also depends on the spectral content of the signal. The rilling study presents the technical aspects of the EMD implementation and makes the five-step algorithm given by the following [3]:

a) Extract the extreme of the signal $x(t)$.

b) Deduce an upper envelope emax ( $t$ ) (resp. lower emin (t)) by interpolation of the maxima (resp. minima).

c) Define a local average $m(t)$ as the sum of the half-envelopes by the expression:

$$
m(t)=\left(e_{\max }(t)+e_{\min }(t)\right) / 2
$$

d) Deduce a local detail $\mathrm{dJ}(\mathrm{t})=\mathrm{IMFJ}(\mathrm{t})$ by the expression:

$$
d(t)=x(t)-m(t)
$$

e) The iteration is given by the expression (1).

The first IMF contains the terms of higher frequencies and contains the following terms of decreasing frequency up to forward only a residue of low frequency.

\subsection{EEMD}

The ensemble EMD method has been proposed to overcome mode mixing problem existing in EMD technique [8]. The EMD technique allows giving all solutions that give the true IMF by repeating the decomposition processes. The procedure of the EEMD method is given as follow:

Step 1: Add white noise with predefined noise amplitude to the signal to be analyzed.
Step 2: Use the EMD method to decompose the newly generated signal.

Step 3: Repeat the above signal decomposition with different white noise, in which the amplitude of the added white noise is fixed.

Step 4: Calculate the ensemble means of the decomposition results as final results.

The signal $\mathrm{x}(\mathrm{k})$ is decomposed into a finite number of intrinsic mode functions (IMFs) and a residue.

$$
x(k)=\sum_{i=1}^{n} \widehat{c}_{i}+\widehat{r}
$$

Where $\mathrm{n}$ represents the number of the IMFs, $\hat{c}_{i}$ is the ith IMF that is the ensemble mean of the corresponding IMF obtained from all of the decomposition processes and $\hat{r}$ is the mean of the residues from all of the decomposition processes.

\subsection{CEEMD Algorithm}

Although EEMD alleviates the effect of mode mixing, noise will remain in the corresponding $\operatorname{IMF}(\mathrm{s})$ if the ensemble number is small. To ensure a noise-free IMF, a CEEMD algorithm [11-13] is introduced as follows:

(1) Add positive and negative white noise $\varepsilon^{+,-}(t)$ into the targeted signal $x(t)$, and construct two new data sets $x^{+}(t)$ and $x^{-}(t)$.

$$
\begin{aligned}
& x^{+}(t)=x(t)+\varepsilon^{+}(t) \\
& x^{-}(t)=x(t)+\varepsilon^{-}(t)
\end{aligned}
$$

(2) Repeat Step 1, and decompose each new data $x^{+}(t)$ and $x^{-}(t)$ using the EMD algorithm;

(3) Obtain two sets of IMFs for the $x^{+}(t)$ and $x^{-}(t)$ signals; (4) Obtain the decomposed result by averaging the $I M F_{k}^{i}$ in Equation (11), where $I M F_{k}^{i}$ represents the i-th IMF of the kth iteration.

$$
\overline{I M F}_{1}(n)=\frac{1}{I} \sum_{i=1}^{l} \overline{I M F_{k}^{i}}(n)
$$

\subsection{Complete Ensemble Empirical Mode of Decomposition with adaptive noise (CEEMDAN)}

To overcome mode mixing problem in EMD we came to EEMD. We overcome the problem of mode mixing but we have another one came into picture. The problem in EEMD is more number of iterations is required which makes the process robust. And also reconstructed signal includes residual noise. So we have new method called CEEMD, provides an exact reconstruction of original signal and better separation of modes with low computation cost. In this method first mode $\overline{I M F}_{1}(n)$ is obtained same way as in EEMD. Then compute first EMD mode over an ensemble of r1 (n) plus different realizations of a given noise obtaining $\overline{I M F}_{2}(n)$ by 
averaging. Here $\mathrm{Ej}$ [.] operator provide $\mathrm{jth}$ mode obtained by EMD. wi is the white noise[12-14]. The steps of CEEMDAN decomposition are as follows:

1. Decompose $x(n)+w_{0} \varepsilon^{i}(n)$ to obtain the first mode by using:

$$
\overline{\operatorname{IMF}}_{1}(n)=\frac{1}{I} \sum_{i=1}^{l} \overline{I M F_{k}^{i}}(n)
$$

Where $w_{0}$ is the amplitude of the added white noise, and $\varepsilon(t)$ is the white noise with unit variance

2. Compute the difference signal:

$$
r_{1}(n)=x(n)-\overline{I M F}_{1}(n)
$$

3. Decompose $r_{1}(n)+w_{1} E_{1}\left(\varepsilon^{i}(n)\right)$, to obtain the first mode and define the second mode by

$$
\overline{I M F}_{2}(n)=\frac{1}{I} \sum_{i=1}^{l} E_{1}\left(r_{1}(n)+w_{1} E_{1}\left(\varepsilon^{i}(n)\right)\right)
$$

For $\mathrm{k}=2, \ldots, \mathrm{K}$, calculate the $\mathrm{k}$-th residue and obtain the first mode. Define the $(\mathrm{k}+1)$-th mode as follows:

$$
\overline{I M F}_{k+1}(n)=\frac{1}{I} \sum_{i=1}^{l} E_{1}\left(r_{k}(n)+w_{k} E_{k}\left(\varepsilon^{i}(n)\right)\right)
$$

where $E_{1}$ is a function to extract the $\mathrm{j}$-th IMF decomposed by EMD

4. Continue this process until residue no longer feasible. Final residue

$R(n)=x(n)-\sum_{k=1}^{k} \overline{I M F}_{k}$

So the given signal can be expressed as

$$
x(n)=R(n)+\sum_{k=1}^{k} \overline{I M F}_{k}
$$

These techniques are tested on MIT-BIH (Massachusetts Institute of Technology Beth Israel Hospital) Arrhythmia database [11].

\section{BIOMEDICAL SIGNALS}

The biomedical signals such as EMG, EEG and ECG are nonstationary and/or nonlinear. The signal used in this work is electrocardiogram; the abnormal signals are obtained from the patients with a CU Ventricular Tachyarrhythmia, Malignant Ventricular and ventricular arrhythmias. The anomaly of CU Ventricular Tachyarrhythmia has a misalignment of the third QRS complex. The figure 2 shows this abnormal signal [15].

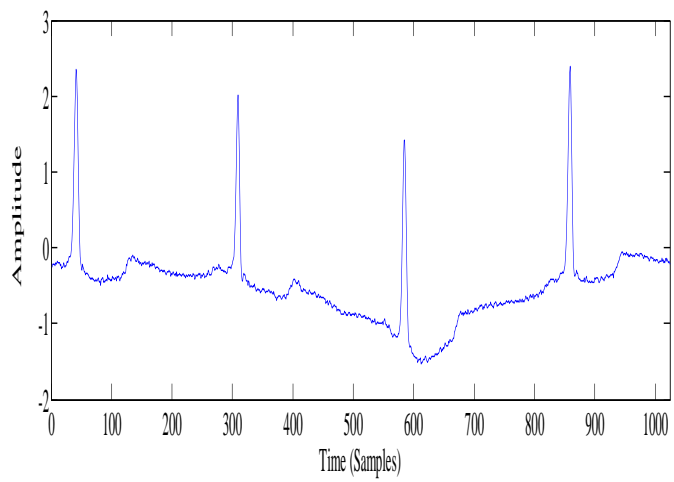

Fig. 2 Abnormal signal CU Ventricular Tachyarrhythmia.

The ventricular arrhythmias manifest as gross distortions of the beat. The QRS complexes are wide and abnormal, while the Twave has disappeared. The signal seems to have an irregularity and is changing over time. The figure 3 shows this anomaly:

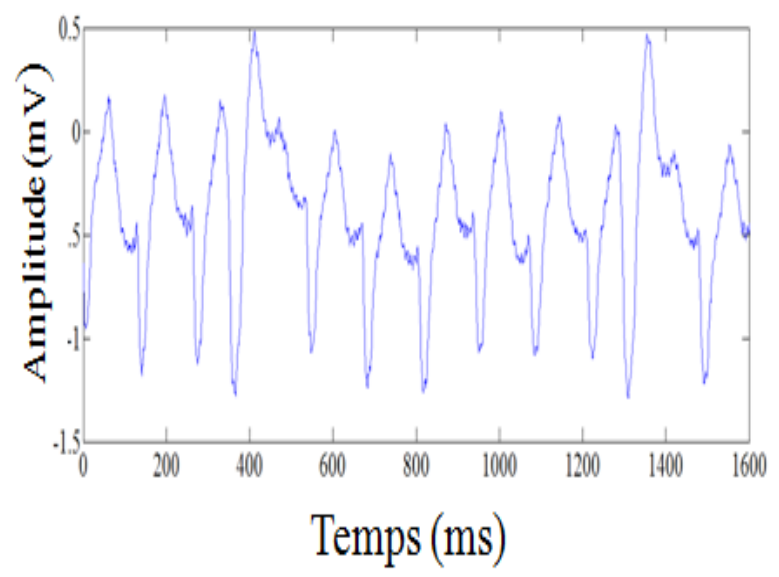

Fig. 3 Abnormal signal ventricular arrhythmias

We compare the performance of the CEEMDAN technique quantitatively with respect to the other techniques based on two metrics: Mean Square Error (MSE) and Percent Root Mean square Difference (PRD). The MSE and PRD are used to evaluate the quality of the information which is preserved in the denoised ECG signal.

MSE and PRD are computed as follows:

$$
\begin{aligned}
M S E & =\frac{1}{N} \sum_{n=1}^{N}(x(n)-\bar{x}(n))^{2} \\
P R D & =\sqrt{\frac{\sum_{n=1}^{N}(x(n)-\bar{x}(n))^{2}}{\sum_{n=1}^{N} x^{2}(n)}} * 100
\end{aligned}
$$

Where $\mathrm{x}(\mathrm{n})$ is the original ECG signal, $\bar{x}(n)$ denotes the reconstruction of the ECG signal and $\mathrm{N}$ is the number of ECG samples used.

\section{RESULTS AND DISCUSSION}

The useful information in the normal and abnormal ECG signals is revealed via $\mathrm{P}$ and $\mathrm{T}$ waves and QRS complex features. The relevant diagnostic information is given by localization of the (P, T waves and QRS complex), duration, amplitudes and shapes determined. During the recording of ECG signals, the artifacts can be interfered with these non- 
stationary signals. In this section, we present two real cases of noise that attack the abnormal signal and perturb the morphology of the PQRST complex; implying a bad diagnosis of heart. These types of noise degrade the quality of the ECG signals; this quality depends on the frequency resolution and the morphology of the waves ( $\mathrm{P}$ and $\mathrm{T}$ ) and QRS complex that contain the important information. In this section, the denoising techniques (CEEMDAN, EEMD and EMD) are applied on abnormal signal by adding the types of noise such as Muscle (EMG) Artifacts (ma) and Electrode Motion (EM); Electrode motion artifact is generally considered as the most troublesome since it can mimic the appearance of ectopic beats and cannot be removed easily by simple filters, as can noise of other types. The advantage of CEEMDAN technique demands less than half of the iterations as the EEMD technique [12-14]. The EEMD gives better results than EMD technique, but consume the time. To overcome this problem; we use the CEEMDAN technique [12-14]. The CEEMDAN technique ameliorates local mean estimation in noisy signals.

Table 1 and figures (4 and 5) present the MSE and PRD of the abnormal signals noised with the Muscle (EMG) artifact (MA). Table 2 and figures (6 and 7) present the MSE and PRD of the abnormal signal noised with the Electrode Motion (EM). The CEEMDAN technique shows the performance for real noises compared to EEMD and EMD methods; the obtained results in the tables show the smallest values of the MSE and PRD obtained by the CEEMDAN technique. These results evaluate the quality of the information which is preserved in the denoised ECG signal compared to other techniques which are usually used in ECG signal denoising technique. The obtained results show the effectiveness of the CEEMDAN technique and its capability of extracting useful information from ECG signals affected by noise as compared to other techniques used in this work such as EEMD and EMD.

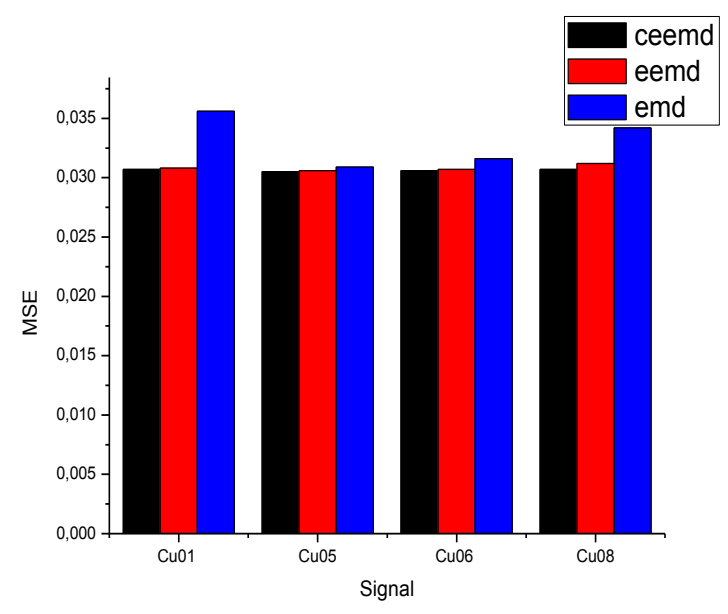

Figure 4: Comparison of PRD obtained by different denoising methods (The noise added is Muscle EMG).

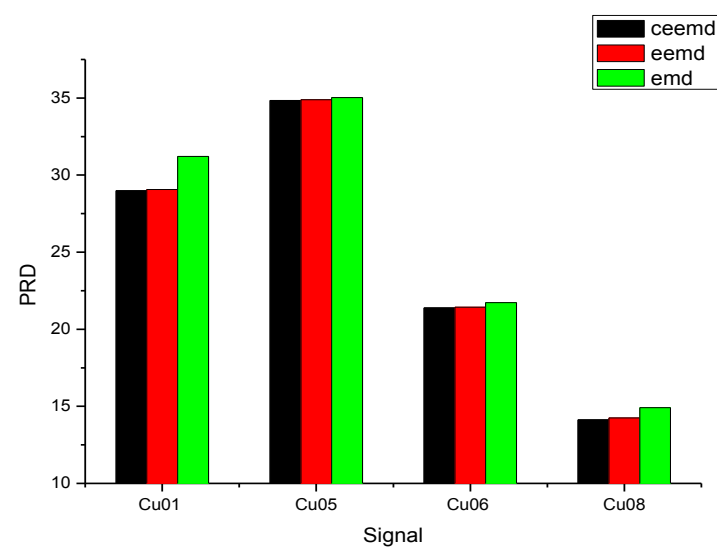

Figure 5 Comparison of PRD obtained by different denoising methods (The noise added is Muscle EMG). Table 1: Comparison of the MSE and PRD obtained by using different technique (the noise added is Muscle EMG)

\begin{tabular}{|l|l|l|l|l|l|l|}
\cline { 2 - 7 } \multicolumn{2}{c|}{} & \multicolumn{3}{c|}{ MSE } & \multicolumn{3}{c|}{ PRD } \\
\hline signal & ceemdan & eemd & emd & ceemdan & eemd & emd \\
& & & & & & \\
\hline Cu01 & 0.0307 & 0.0308 & 0.0356 & 28.9853 & 29.0597 & 31.2094 \\
Cu05 & 0.0305 & 0.0306 & 0.0309 & 34.8396 & 34.8904 & 35.0320 \\
Cu06 & 0.0306 & 0.0307 & 0.0316 & 21.3935 & 21.4275 & 21.7263 \\
Cu08 & 0.0307 & 0.0312 & 0.0342 & 14.1213 & 14.2522 & 14.9106 \\
& & & & & & \\
\hline
\end{tabular}

Table 2: Comparison of the MSE and PRD obtained by using different technique (the noise added is Electrode Motion)

\begin{tabular}{|c|c|c|c|c|c|c|}
\cline { 2 - 7 } \multicolumn{1}{c|}{} & \multicolumn{2}{c|}{ MSE } & \multicolumn{2}{c|}{ PRD } \\
\hline $\begin{array}{c}\text { Signals Malignant } \\
\text { Ventricular } \\
\text { arrhythmias }\end{array}$ & ceemdan & eemd & emd & ceemdan & eemd & emd \\
\hline 423 & & & & & 257.1040 & 257.2533 \\
424 & 0.1743 & 0.1745 & 0.1747 & 256.9080 & 231.7286 & 170.2131 \\
\hline 425 & 0.1743 & 0.1744 & 0.1750 & 231.6059 & 169.9687 & \\
\hline
\end{tabular}




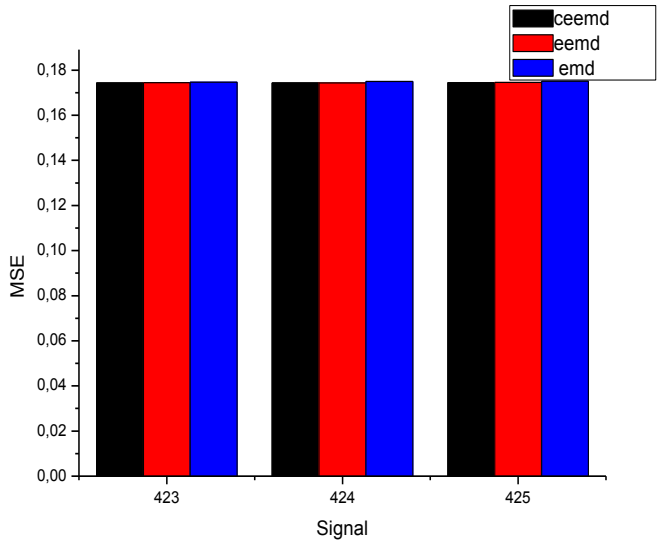

Figure 6: Comparison of PRD obtained by different denoising methods (The noise added is the Electrode Motion).

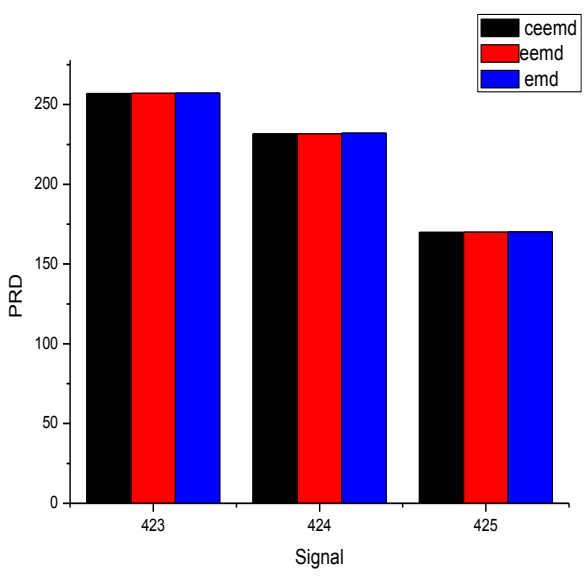

Figure 7: Comparison of PRD obtained by different denoising methods (The noise added is the Electrode Motion)

\section{CONCLUSION}

In this research, CEEMDAN, EEMD and EMD techniques are applied on biomedical signals; the ECG subjects used as cu tachyarrhythmia ventricular, Malignant Ventricular arrhythmias in order to eliminate the effects of noise which masks the useful information. The real noises used are Muscle Artifacts and Electrode Motion. The main advantage of CEEMDAN techniques is the fact that they do not make any prior assumption about the data being analyzed. The CEEMDAN technique shows the cancellation of artifacts of abnormal signal which are due to different noises. The obtained result shows that CEEMDAN would be a useful technique to remove the nonlinear interferences from the ECG signal. In future works, we focus on the time-frequency analysis using Hilbert-Huang transform by comparing the results of CEEMDAN and EEMD techniques.

\section{REFERENCES}

[1] P. de Chazal, Maraia O' Dwyer, Richard B. Reilly, "Automatic classification of heartbeats using ECG morphology and heartbeat interval features", IEEE Transactions on Biomedical Engineering 51 (7) pp. 11961206, 2004.

[2] G Bortolan, Christian Brohet, Sergio Fusaro, "Possibilities of using neural networks for ECG classification", Journal of Electrocardiology 29 Suppl:10-16, 1996.
[3] N. E. Huang, Z. Shen, S. R. Long, M. C. Wu, E. H. Shih, Q. Zheng, C. C. Tung and H. H. Liu, The empirical mode decomposition method and the Hilbert spectrum for nonstationary time series analysis, Proc. Roy. Soc. London 454A (1998) 903-995.

[4] S. Elouaham, R. Latif, B. nassiri, A. Dliou, M. Laaboubi, F. Maoulainine, Analysis electrocardiogram signal using ensemble empirical mode decomposition and timefrequency techniques. International Journal of Computer Engineering \& Technology (IJCET), Vol 4, N 2, 2013, 275 289.

[5] P. Flandrin, G. Rilling and P. Goncalves, Empirical mode decomposition as a filter bank, IEEE Signal Process. Lett. 11 (2004) 112-114.

[6] S. Elouaham, R. Latif, A. Dliou, M. Laaboubi, F. M. R. Maoulainine, Biomedical Signals Analysis Using the Empirical Mode Decomposition and Parametric and non Parametric Time-Frequency Techniques, International Journal on Information Technology (IREIT), Vol. $1 \mathrm{~N} .1$, 2013, 1-10.

[7] Azzedine Dliou; Rachid Latif; Mostafa Laaboubi; Fadel Mrabih Rabou Maoulainine; Samir Elouaham, "Timefrequency analysis of a noised ECG signals using empirical mode decomposition and Choi-Williams techniques", International Journal of Systems, Control and Communications, Vol. 5 No. 3/4, pp.231:245, 2013.

[8] S. Elouaham, R.Latif, B.Nassiri, A. Dliou, M. Laaboubi, F. Maoulainine, "Analysis Electroencephalogram signals using ANFIS and Periodogram techniques". International Review on Computers and Software (I.RE.CO.S.), Vol. 8, n.12. pp 2959: 2966, 2013.

[9] A.N. Akansu, W.A. Serdijn, and I.W. Selesnick, Wavelet Transforms in Signal Processing: A Review of Emerging Applications, Physical Communication, Elsevier, vol. 3, issue 1, pp. 1-18, March 2010.

[10] Akansu, Ali N.; Haddad, Richard A. (1992), Multiresolution signal decomposition: transforms, subbands,

[11] Yeh, J.-R.; Shieh, J.-S.; Huang, N.E. Complementary ensemble empirical mode decomposition: A novel noise enhanced data analysis method. Adv. Adapt. Data Anal. 2010, 2, 135-156.

[12] Torres, M.E.; Colominas, M.A.; Schlotthauer, G.; Flandrin, P. A complete ensemble empirical mode decomposition with adaptive noise. In Proceedings of 2011 IEEE International Conference on Acoustics, Speech and Signal (ICASSP), Prague, Czech, 22-27 May 2011; pp. 41444147.

[13] He, X.; Goubran, R.A.; Liu, X.P. Ensemble Empirical Mode Decomposition and adaptive filtering for ECG signal enhancement. In Proceedings of 2012 IEEE International Symposium on Medical Measurements and Applications (MeMeA), Budapest, Hungary, 18-19 May 2012; pp. 1-5.

[14] Marcelo A. Colominas, Gastón Schlotthauer, María E. Torres 'Improved complete ensemble EMD: A suitable tool for biomedical signal processing'Biomedical Signal Processing and Control Volume 14, November 2014 Pages 19-29.

[15] Physiobank, Physionet, Physiologic signal archives for biomedical research. 